\section{The ultrastructural changes of tendon axonal profiles of medial rectus muscles according to duration in patients with intermittent exotropia}

S-H Kim', YA Cho' ${ }^{1}$ C-H Park ${ }^{2}$ and C-S Uhm²
${ }^{1}$ Department of Ophthalmology, Korea University College of Medicine, Seoul, Korea

${ }^{2}$ Department of Anatomy, Korea University College of Medicine, Seoul, Korea

Correspondence: S-H Kim, Department of Ophthalmology, Korea University College of Medicine,

Ansan Hospital, 516 Gojan-dong, Ansan, Gyunggi-do 425-707,

Republic of Korea

Tel: + 8231412 5160;

Fax: +82 314148930

E-mail: ansaneye@

hanmail.net

Received: 14 December 2007

Accepted in revised form: 11 April 2008

Published online: 23 May 2008

\begin{abstract}
Purpose The goal of this study was to investigate the ultrastructural changes of tendon axonal profiles of medial recti in patients with intermittent exotropia at different ages. In addition, we compared the patterns of degeneration with those of secondary exotropia over time.

Methods Thirteen patients, with different ages, with exotropia who had undergone surgery were included in this study and divided into two groups. Eight patients had intermittent or constant exotropia; their age ranged from 6 to 45 years and they had exotropia since childhood without amblyopia, these patients were assigned to group $A$. The other five patients with sensory exotropia ranged in age from 15 to 52 years; they did not have exotropia until a visual insult and had poor vision in one eye, these patients were assigned to group B. All patients had the medial recti resected $(3-5.5 \mathrm{~mm})$ to obtain tissue samples. All specimens were examined with an electron microscope.

Results Schwann cell degeneration was observed with increased neurofilament density, axonal vacuoles and hydropic swelling of the Schwann cells in two patients less than 10 years of age in group $A$. The other six patients were more than $\mathbf{1 0}$ years of age in group $A$, and it was not possible to identify the tendon axonal profiles or neural structures in the medial recti specimens of these patients. For group B, all patients had intact proprioceptor structures including Schwann cells. However, the collagen diameter
\end{abstract}

decreased and density increased within the capsule according to the duration of exotropia. Conclusion Schwann cell degeneration of tendon proprioceptors in the medial rectus might induce the degeneration of proprioceptors in patients with intermittent exotropia over time.

Eye (2008) 22, 1076-1081; doi:10.1038/eye.2008.133; published online 23 May 2008

Keywords: tendon axonal profiles; proprioceptor; Schwann cell degeneration; intermittent exotropia; secondary exotropia; ultrastructural changes according to age

Introduction

The most common type of exodeviation seen in an ophthalmology practice is intermittent exotropia. This is the most challenging type of exodeviation to be cured in the long term. Exodeviations may be controlled by fusion mechanisms and are labelled as exophoria, intermittent exotropia, or constant exotropia. ${ }^{1}$ We have previously reported on the ultrastructural findings of tendon axonal profiles in the medial rectus muscles of patients with intermittent exotropia. These patients had unexpected Schwann cell degeneration. This was unexpected because Schwann cells are not considered primary structural elements that participate in conduction but rather as supporting neural structures for axonal growth. We previously suggested a future study for clarifying the role and consequences of Schwann cell degeneration in intermittent exotropia. ${ }^{2}$ 
The purpose of this study was to evaluate patients presenting with intermittent exotropia at various ages and to document the ultrastructural changes of the axonal profiles from Schwann cell degeneration at the distal myotendons of the medial rectus muscles over time. In addition, we compared these findings with acquired sensory exotropia, which can be caused by the loss of vision in one eye and decreased medial rectus tonicity, to determine whether there were alterations in the Schwann cells. ${ }^{3}$ The results of these comparisons might help determine whether the Schwann cell degeneration found in the medial recti tendon axonal profiles with intermittent exotropia are primary changes or secondary to the consequent loss of tonicity.

\section{Patients and methods}

We studied 13 patients with exotropia and divided them into two groups. Group A consisted of eight patients with intermittent or constant exotropia; they were between 6 and 45 years of age and had exotropia since childhood, without amblyopia. The other five patients with sensory exotropia, between the ages of 15 and 52 years, with exotropia and poor vision in one eye and a history of a visual insult, before developing exotropia, were assigned to group B as a control. All patients provided informed consent. This study was conducted in accordance with the tenets of the Declaration of Helsinki. All patients underwent surgery including unilateral medial rectus resection. The resected medial recti specimens $(3-5.5 \mathrm{~mm})$ were evaluated (Tables 1 and 2).

Immediately after resection, the specimens were fixed for $4 \mathrm{~h}$ at $4{ }^{\circ} \mathrm{C}$ in a mixture of $2 \%$ glutaraldehyde and $2 \%$ paraformaldehyde in $0.1 \mathrm{M}$ sodium phosphate buffer ( $\mathrm{pH}$ 7.3)

The tissues were postfixed with $1 \% \mathrm{OsO}_{4}$ at room temperature in the same buffer for $2 \mathrm{~h}$, then dehydrated in a graded series of ethanol; then they were embedded in Poly-bed 812. Next, we analysed in steps a series of semi-thin cross sections $(1 \mathrm{~mm})$ that were stained with toluidine blue. Proceeding from the proximal to the distal ends of the specimens, each block was scanned using a light microscope for gross inspection of the muscle fibres, tendon collagen tissue and the location of neural structures. Ultrathin transverse sections were cut and

Table 1 Preoperative evaluation and the surgical procedure of group A patients

\begin{tabular}{lccll}
\hline Patient no. & $\begin{array}{c}\text { Age } \\
\text { (years) }\end{array}$ & $\begin{array}{c}\text { Preoperative alignment } \\
(P D, D / N)\end{array}$ & Phase & $\begin{array}{l}\text { Procedure } \\
(\mathrm{mm})^{\mathrm{a}}\end{array}$ \\
\hline 1 & 6 & $35 / 30$ & Intermittent & R\&R (3.5) \\
2 & 7 & $35 / 35$ & Constant (R) & BMR resection (4/3.5) \\
3 & 16 & $75 / 80$ & Constant & Four muscle surgery (3.5/3) \\
4 & 18 & $20 / 18$ & Constant (R) & LMR resection (4) \\
5 & 33 & $35 / 35$ & Constant (R) & LMR resection (5.5) \\
6 & 38 & $60 / 60$ & Constant & Three muscle surgery (3.5) \\
7 & 41 & $70 / 85$ & Constant & Four muscle surgery (5/4.5) \\
8 & 45 & $100 / 100$ & Constant & Four muscle surgery (5/5) \\
\hline
\end{tabular}

a Resection amount of medial rectus muscles.

$\mathrm{R}$, recurrent; $\mathrm{D} / \mathrm{N}$, measured angle at distant and near; $\mathrm{R} \& \mathrm{R}$, unilateral lateral rectus recession and medial rectus resection; Three muscle surgery, bilateral lateral rectus recession and unilateral medial rectus resection; Four muscle surgery, bilateral lateral rectus recession and bilateral medial rectus resection.

Table 2 Preoperative evaluation and surgical procedure of group B patients

\begin{tabular}{|c|c|c|c|c|}
\hline Patient no. & Cause & $\begin{array}{l}\text { Preoperative alignment } \\
(P D, D / N)\end{array}$ & $\begin{array}{c}\text { Age/duration } \\
\text { (years) }^{\mathrm{a}}\end{array}$ & $\begin{array}{l}\text { Procedure } \\
(\mathrm{mm})\end{array}$ \\
\hline 1 & Congenital glaucoma & $30 / 40$ & $16 / 15$ & R\&R (3) \\
\hline 2 & Optic neuropathy & $30 / 30$ & $24 / 20 ?^{\mathrm{a}}$ & $\mathrm{R} \& \mathrm{R}(3.5)$ \\
\hline 3 & Optic neuropathy & $35 / 35$ & $38 / 30 ?^{a}$ & R\&R (3) \\
\hline 4 & $\begin{array}{l}\text { Corneal opacity } \\
\text { (epithelial ingrowth after LASIK) }\end{array}$ & $30 / 35$ & $33 / 1$ & $\mathrm{R} \& \mathrm{R}$ (3.5) \\
\hline 5 & Surgical aphakia & $45 / 45$ & $52 / 3$ & $R \& R(4)$ \\
\hline
\end{tabular}

a Patients did not remember the onset of exodeviation exactly.

$R \& R$, unilateral recession of lateral rectus and resection of medial rectus; $D / N$, measured angle at distant and near. 
mounted on cupper grids and stained with uranyl acetate and lead citrate, and then they were evaluated by a neuroanatomist (C-SU) under an electron microscope (Hitachi, H-600).

\section{Results}

Eight patients in group A had an intermittent (one patient) and constant exodeviation, of more than $20 \mathrm{PD}$, at a $6 \mathrm{~m}$ distance target. The mean amount of exotropia, at a distance was 54 PD (range 20-100). Three patients had recurrent exodeviation. All patients underwent surgery including medial rectus resection. The total number of MR specimens was 12. The amount of resected material from the medial recti was $3-5.5 \mathrm{~mm}$ (Table 1). Group B consisted of five patients with constant exodeviation, of more than $30 \mathrm{PD}$, at a $6 \mathrm{~m}$ distance target, with poor vision in one eye. The visual acuity of deviating eye, among all group B patients, was less than 20/200. Two patients suffered from an optic neuropathy, and the other visual insults were congenital glaucoma, ocular trauma, and a corneal opacity, due to an epithelial ingrowth after LASIK (Table 2). All patients underwent unilateral $\mathrm{R}$ and $\mathrm{R}$ (recession and resection). The amount of the resected medial recti was 3-4 mm (Table 2).

We identified nerve fibres in only two patients from group A (patients 1 and 2); they were both under the age of 10 years. We were not able to find any neural structures in the resected muscle specimens of the other six patients (patient nos.: 3-8). In two group A patients, increased fibrillar elements such as neurofilaments and hydropic swelling of Schwann cells were frequently noted, but the overall integrity of the myelin sheaths and axons were relatively well preserved. In addition, vacuoles in the axoplasm were noted (Figures 1 and 2). The overall impression was that the structural integrity of the proprioceptors was not well maintained in group A. On the other hand, for the patients in group B, nerve fibres were easily identified in all patients. We observed the intact typical structure of the proprioceptors. The capsules and Schwann cells were all intact. However, in the patients with long-standing exotropia, more than 10 years of age, myelinated axons were not detected. The collagen fibrillar density within the capsule increased and the diameter decreased; the collagen appeared to be immature fibres. Fibroblasts were sparse and the organelles within the capsules were separated from the capsules themselves (Figure 2a-c). By contrast, in the patients with recently developed exotropia, myelinated axons were identified but vacuoles were detected in them. There was also an increased number of immature collagen, and fibroblasts were sparse (Figure 2d). These findings suggested degeneration causing the loss of tonus in the medial recti of patients with acquired sensory exotropia.

\section{Discussion}

Intermittent exotropia is commonly found in the Middle East, subequatorial Africa, and Asia. ${ }^{4}$ Most cases of exodeviation have no known cause. Controversy exists as to whether exodeviation is caused by anatomic and mechanical factors within the orbit or by innervation abnormalities, excessive tonic divergence innervation, or reduced convergence innervation. ${ }^{5}$

Monocular visual loss frequently results in an exodeviation called sensory exotropia, which is caused by the loss of tonic convergence. ${ }^{3}$ We previously speculated that the agonist of intermittent exotropia might be the medial recti, caused by problems with convergence. ${ }^{2}$ Axonal profiles of the medial recti showed striking differences only between intermittent and infantile exotropia; the Schwann cells observed in these cases of intermittent exotropia were degenerated. These findings are supported by the numerous clinical classifications of intermittent exotropia according to the patterns of exodeviation relative to distance and near fixation. ${ }^{6,7}$ The degree of convergence tonus is different when basic convergence insufficiency and

pseudodivergence types are compared. In addition, the excess type of true divergence, which reflects excessive tonic divergence innervation, is rare. ${ }^{8}$ Therefore, the documentation of the pathological findings of the medial recti tendon axonal profiles, in various types of exotropia, provides important information.

Corsi et $a l^{9}$ studied the ultrastructure of sensorial receptors located at the myotendinous junctions of 11 subjects with congenital esotropia. Important alterations of these receptors were observed; the patients with strabismus had abnormal reception and transmission of proprioceptive information. Trotter and co-workers ${ }^{10,11}$ investigated the role of proprioceptors in the pathogenesis of strabismus. They demonstrated abnormal depth perception and a reduced proportion of binocular cells in kittens that had their proprioceptive afferents interrupted by cutting the unilateral ophthalmic branches of the trigeminal nerve during the critical period of development. This finding supports the hypothesis that some disturbance of ocular proprioception may be involved in the pathogenesis of congenital strabismus.

The myelin sheath and axon were well preserved in intermittent exotropes under the age of 10 years. However, the structural integrity of proprioceptors was not well maintained. There were no neuronal components of the proprioceptors identified in patients 

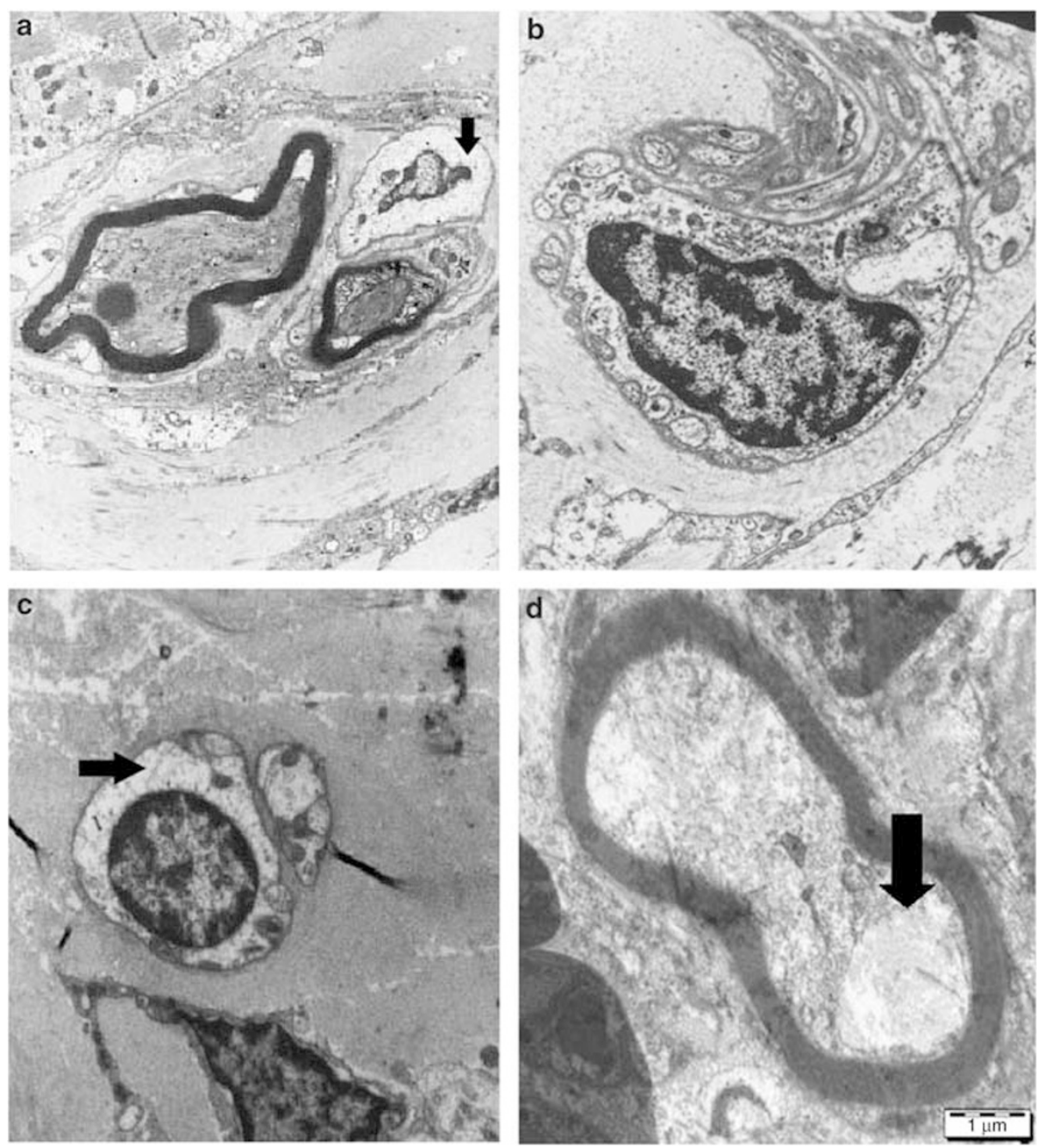

Figure 1 Degenerative findings of tendon axonal profiles according to seniority in intermittent exotropia (group A): no neural structures could be found in patients nos. $3-8$, who were over 10 years of age and in the constant phase. (a, b) The findings of a 6-yearold patient with the intermittent phase (no. 1): increased fibrillar elements in the axonal cytoplasm were observed, but the overall integrity of myelin, the sheath, and axon were relatively well preserved. Hydropic swelling of the Schwann cell cytoplasm (arrow) is noted (a), but normal Schwann cells are also seen (b); $\times 15000$ (a), $\times 6000$ (b). (c, d) The findings of a 7-year-old patient with the constant and recurrent phases (no. 2): hydropic swelling of Schwann cell cytoplasm (arrow) was also noted (c), but vacuoles in the axoplasm (arrow) were also noted (d). The axonal integrity was intact, but structural integrity of the proprioceptors was not detected; $\times 15000$.

with long-standing exotropia, more than 10 years of age in group A. Richmond et $a l^{12}$ reported fine nerve components in only three specimens out of 12 , in patients aged 9 months to 10 years; the specimens were obtained during the resection procedures for the surgical correction of strabismus. However, they did not clarify the types of strabismus. Martini ${ }^{13}$ suggested that mutant Schwann cells caused a significant reduction of the axonal caliber, they increased the density of neurofilaments, and locally slowed axonal transport. Ultimately, this resulted in axonal degeneration. Our previous study illustrated these consequences. ${ }^{2}$ Our results show the results of Schwann cell degeneration; the initial pathological events affecting the Schwann cells lead to the degeneration of axons, and perhaps affect the integrity of proprioceptors. Clinically, all of the patients with constant exotropia, in this study, had no structural evidence of proprioceptors in the medial recti myotendon. Future study is needed to clarify the relationship between the phase (intermittency) of intermittent exotropia and the pathological findings of proprioceptors with a larger group of patients.

Hertle $e t a l^{14}$ described the findings of the enthesial section of the medial rectus muscle in a 19-year-old patient with sensory exotropia. In this patient, the extracellular matrix consisted mainly of collagen fibrils with periodicity and diameters from 50 to $60 \mathrm{~nm}$. The investigators could not determine why this patient had 

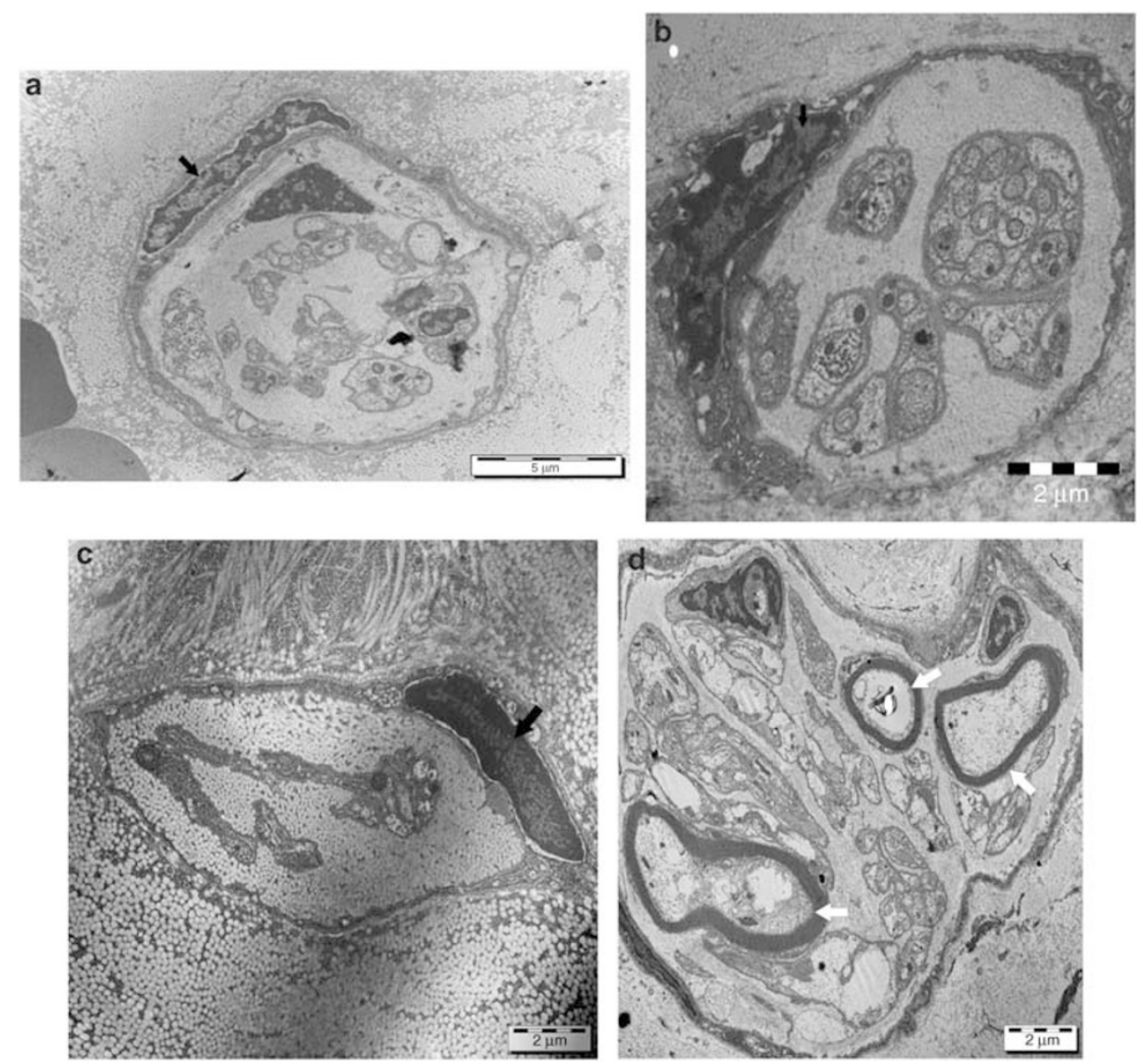

Figure 2 Various findings according to disease duration for sensory exotropia (group B): the formation of the tendon axonal profiles was encircled by the capsule. All Schwann cells (black arrows) were intact. Inside of the capsule, immature collagen density increased according to the duration of exodeviation. Fibrocytes were sparse. $(a-c)$ The findings of patient nos. 1-3. Three myelinated nerve fibres inside the capsule (white arrows) are seen in the picture of patient 4 with recent onset exodeviation (d).

anomalous findings of collagen density and size in the extracellular matrix in proprioceptors. We observed similar anomalous findings in the extracellular matrix, in a series of sensory exotropes of variable duration. The longer the duration of sensory exotropia the greater the increase in the collagen density and decrease of the collagen size in the proprioceptors. These findings might represent secondary changes that follow the loss of tonic convergence in the medial rectus muscles. By contrast, the findings noted in the patients with intermittent exotropia suggest primary changes of Schwann cell degeneration. The family history of patients, with intermittent exodeviations, indicates a hereditary basis for the misalignment in some cases. ${ }^{15}$ Schwann cellrelated genetic studies of intermittent exotropes are planned in the future.

It is not yet possible to conclude that proprioceptive alterations and degeneration represent the etiological factors that cause intermittent exotropia or if these changes are due to altered innervation. Animal studies have shown that afferent fibres from the palisade endings run with the III, IV, and VI cranial nerves before joining the ophthalmic division of the trigeminal nerve, and terminate in the ipsilateral spinal trigeminal nuclei via the trigeminal ganglion. From these brain stem nuclei, there are numerous connections to the areas involved in visual and ocular motor control. ${ }^{16-20}$ Therefore, although the course and subsequent connections of afferent fibres from extraocular muscles in humans are not fully understood, architectural derangement of sensorial receptors may be related to the alteration of the sensory nerve supply as well as the motor output.

The results of this study showed that the ultrastructure of the tendon axonal profiles, of intermittent exotropia, degenerate over time and the degeneration might be caused by primary changes that differ from secondary acquired sensory exotropia. These findings may help improve our understanding of the pathogenesis of intermittent exotropia. 


\section{References}

1 Plager DA. Strabismus Surgery: Basic and Advanced Strategies. Oxford University Press: New York, 2004, pp 17-18.

2 Kim SH, Yi ST, Cho YA, Uhm CS. Ultrastructural study of extraocular muscle tendon axonal profiles in infantile and intermittent exotropia. Acta Ophthalmol Scand 2006; 84 182-187.

3 Von Noorden GK. Binocular Vision and Ocular Motility, 6th ed. CV Mosby: St Louis, 2002, pp 30-31.

4 Von Noorden GK. Binocular Vision and Ocular Motility, 6th ed. CV Mosby: St Louis, 2002; 342-343.

5 Von Noorden GK. Binocular Vision and Ocular Motility, 6th ed. CV Mosby: St Louis, 2002; 356-358.

6 Burian HM. Exodeviation: their classification, diagnosis, and treatment. Am J Ophthalmol 1966; 62: 1161.

7 Kushner BJ. Exotropic deviation: a functional classification and approach to treatment. Am Orthopt J 1988; 38: 81-93.

8 Von Noorden GK. Binocular Vision and Ocular Motility, 6th ed. CV Mosby: St Louis, 2002; 361.

9 Corsi M, Sodi A, Salvi G, Faussone-Pellegrini MS. Morphological study of extraocular muscle proprioceptor alteration in congenital strabismus. Ophthalmologica 1990; 200: 154-163.

10 Trotter Y, Fregnac Y, Buisseret P. The period of susceptibility of visual cortical binocularity to unilateral proprioceptive deafferentiation of extraocular muscles. J Neurophysiol 1987; 58: 795-815.

11 Graves AL, Trotter Y, Fregnac Y. Role of extraocular muscle proprioception in the development of depth perception in cats. J Neurophysiol 1987; 58: 816-831.
12 Richmond FJ, Johnston WS, Baker RS, Steinbach MJ. Palisade endings in human extraocular muscles. Invest Ophthalmol Vis Sci 1984; 25: 471-476.

13 Martini R. The effect of myelinating Schwann cells on axons. Muscle Nerve 2001; 24: 456-466.

14 Hertle RW, Chan CC, Galita DA, Maybodi M, Crawford MA. Neuroanatomy of the extraocular muscle tendon enthesis in macaque, normal human, and patients with congenital nystagmus. J AAPOS 2002; 6: 319-327.

15 Matsuo T, Yamane T, Ohtsuki H. Heredity vs abnormalities in pregnancy and delivery as risk factors for different types of comitant strabismus. J Pediatr Ophthalmol Strabismus 2001; 38(2): 78-82.

16 Abrahams VC, Rose PK. Projections of extraocular, neck muscle, and retinal afferents to superior colliculus in the cat: their connections to cells of origin to tectospinal tract. J Neurophysiol 1975; 38: 10-18.

17 Baker R, Precht W, Llinas R. Mossy and climbing fiber projections of extraocular muscle afferents to the cerebellum. Brain Res 1972; 38: 440-445.

18 Busisseret P, Maffer L. Extraocular proprioceptive projections to the visual cortex. Exp Brain Res 1977; 28: 421-425.

19 Buisseret-Delmas C, Epelbaum M, Buisseret P. The vestibular nuclei of cat receive a primary afferent projection from receptors in extraocular muscles. Exp Brain Res 1990; 81: 654-658.

20 Donaldson IML, Dixon RA. Excitation of units in the lateral geniculate and contiguous nuclei of the cat by stretch of extrinsic ocular muscles. Exp Brain Res 1980; 38 245-255. 